\title{
The incidence of duplicate genetic testing
}

Douglas L. Riegert-Johnson, $M D^{1}$, Daniela Macaya, $M Q C^{2}$, Timothy W. Hefferon, PhD ${ }^{3}$, and Lisa A. Boardman, $M D^{1}$

\begin{abstract}
Purpose: Duplicate genetic testing (DGT) should give the same results as the initial genetic test. Therefore, DGT is indicated only in the rare instances where the initial results require confirmation. The objective of this study was to determine the incidence of DGT by reviewing TPMT, HFE, and CYP450 2D6 polymorphism testing performed in our institution's laboratories in 2006. A secondary objective was to determine the savings in charges that resulted from a system in place to limit HFE DGT. Methods: A retrospective records review at an academic medical center. Results: The percentage of patients having the same genetic test more than once in 2006 was 3.3\% (253/7710) for TPMT, 0.3\% for HFE (24/7851), and 0.9\% (4/433) for CYP450 2D6 testing. Retail laboratory charges for the DGT identified in 2006 were $\$ 76,728$. To estimate the incidence of DGT over a longer period of time than 2006 , an all-time records review was performed on a subset of internal patients and found the all-time incidence of DGT for TPMT, HFE, and CYP450 2 D6 testing to be $6.9 \%, 1.9 \%$, and $0.9 \%$, respectively. No case of DGT with an appropriate indication for duplicate testing was found. A system in place to decrease HFE DGT is estimated to have saved $\$ 77,479$ in charges for 2006 (95\% Cl, \$35,512-184,015). Conclusions: Indicated DGT is rare and decreasing DGT could result in significant savings. Institutions should consider implementing a systems-based process to limit DGT. Genet Med 2008:10(2):114-116.
\end{abstract}

Key Words: genetic testing, molecular diagnostic techniques, duplicate genetic testing, laboratory techniques and procedures, inappropriate laboratory utilization

Most genetic testing uses DNA extracted from peripheral blood lymphocytes. DNA extracted from these cells rarely changes during a person's lifetime, and a duplicate genetic test should give the same the results as the initial test. Therefore, duplicate genetic testing (DGT) is indicated only in the rare circumstances where the initial results require confirmation. Examples of appropriate indications for DGT include concern for switching of samples or when genetic testing results are unexpected and require confirmation.

This is the first study of the incidence of DGT, specifically the incidence of duplicate HFE, TPMT, and CYP450 2D6 polymorphism genetic testing. Mutations in the HFE gene are associated with the iron overload disorder hemochromatosis. ${ }^{1}$ The TPMT gene codes for the thiopurine $S$-methyl transferase enzyme, which is involved in metabolizing the immunosuppressant drug azathioprine. Clinicians use TPMT testing to identify patients who are poor metabolizers of azathioprine metabolites and are at high risk developing aziothioprine-re-

From the ${ }^{1}$ Mayo Clinic College of Medicine, Division of Gastroenterology, Rochester, Minnesota; ${ }^{2}$ University of Costa Rica, Department of Medical Genetics, San Jose, Costa Rica; and ${ }^{3}$ National Institutes of Health, National Human Genome Research Institute, Bethesda, Maryland.

Douglas L. Riegert-Johnson, MD, Division of Gastroenterology and Hepatology, Mayo Clinic College of Medicine, 200 Second Street SW, Rochester, MN 55905. E-mail: riegertjohnson.douglas@mayo.edu.

Disclosure: The authors declare no conflict of interest.

Submitted for publication September 11, 2007.

Accepted for publication October 18, 2007.

DOI: 10.1097/GIM.0b013e31816166a7 lated side effects. ${ }^{2}$ CYP450 2D6 polymorphisms can predict a patient's response to therapy and the possibility of adverse reactions from antidepressants and other drugs metabolized by the CYP450 2D6 drug metabolism enzyme. ${ }^{3}$

We reviewed all HFE testing and the majority of TPMT and internally referred CYP450 2D6 testing performed in Mayo Clinic laboratories in 2006 to determine the percentage of patients who had the same genetic test performed more than once in 2006. In addition, to determine the incidence of DGT over a longer period of time, we performed an all-time records review on a subset of consecutive patients from inside our institution that had HFE, TPMT, or CYP450 2D6 testing in 2006.

To investigate whether the type of medical practice (academic vs. community) was associated with an increased incidence of DGT, we compared the incidence of DGT for HFE and TPMT testing in internal and external referral groups. The internal sample cohort consisted of patients seen in our institution's academic medical center practice, while the external sample cohort represented an admixture of samples referred from both academic and community-based practices.

During the time this study was performed, the laboratory performing HFE testing had a system in place to limit HFE DGT. This report also includes the performance of this system and recommendations for the management of DGT.

\section{MATERIALS AND METHODS}

Databases of all HFE testing, 84\% of TPMT testing (8000/ 9537), and $49 \%$ of internally referred CYP450 2D6 testing 
(437/884) performed in 2006 by Mayo Clinic laboratories were reviewed for DGT. In addition, consecutive series of patients from inside our institution who had HFE, TPMT, or CYP450 $2 D 6$ testing in 2006 underwent further all-time records review for DGT.

HFE, TPMT, and CYP450 2D6 testing was performed in three separate laboratories. HFE testing comprised testing for the C282Y, H63D, and S65C mutations using Lightcycler ${ }^{\circledR}$ technology. TPMT testing was performed using enzymatic end point or liquid chromatography-tandem mass spectrometry. CYP450 2D6 polymorphism testing was performed using allele-specific primer extension or bead hybridization with fluorescence detection. The Mayo Clinic Institutional Review Board approved this study.

\section{RESULTS}

\section{Duplicate genetic testing}

The percentage of patients having HFE, TPMT, and CYP450 $2 D 6$ testing in 2006 who had a duplicate test in 2006 is shown in Table 1.

The most commonly cited reasons for DGT by laboratory personnel and ordering clinicians were lack of time to adequately review records for previous testing, difficulty in accessing records of previous testing, and lack of understanding by the ordering clinician that DGT will give the same results as the initial test.

There was no significant difference in DGT between the internal or external referral cohorts for TPMT and HFE testing (TPMT $P=0.13$, HFE $P=0.15$, two-tailed $P$ value using Fisher's exact test). We had expected to find a higher rate of DGT in the external referral cohort, as it included samples referred from community practice where physicians were hypothesized to be less adept at genetic testing.

A higher percentage of patients having TPMT testing had duplicate testing than patients having HFE or CYP450 2D6 testing. Many patients had TPMT testing three or more times

\section{Table 1}

Percentage of patients having duplicate HFE, TPMT, and CYP450 2D6 polymorphism genetic testing

\begin{tabular}{lllc}
\hline & & \multicolumn{2}{c}{$\begin{array}{c}\text { Percentage of patients with DGT (patients } \\
\text { with DGT/patients in group) }\end{array}$} \\
\cline { 3 - 4 } Test & Cohort & \multicolumn{1}{c}{ In 2006 only } & At anytime in the past ${ }^{a}$ \\
\hline TPMT & All & $3.3 \%(253 / 7710)$ & - \\
& Internal & $2.5 \%(25 / 996)$ & $6.9 \%(17 / 246)$ \\
& External & $3.4 \%(228 / 6714)$ & - \\
HFE & All & $0.3 \%(24 / 7851)$ & - \\
& Internal & $0.6 \%(4 / 681)$ & $1.9 \%(4 / 207)$ \\
& External & $0.3 \%(20 / 7170)$ & $0.9 \%(4 / 433)$ \\
\hline
\end{tabular}

${ }^{a}$ Incidence of DGT at anytime in the past determined for consecutive series of internal patients only.
Table 2

Distribution of number of TPMT tests per patient in 2006

\begin{tabular}{lcc}
\hline \multirow{2}{*}{$\begin{array}{l}\text { Number of TPMT tests per } \\
\text { patient }\end{array}$} & \multicolumn{2}{c}{ Cohort (number of patients) } \\
\cline { 2 - 3 } & Internal & External \\
\hline 1 & 971 & 6486 \\
2 & 23 & 211 \\
3 & 2 & 11 \\
4 & - & 1 \\
5 & - & 3 \\
6 & - & 1 \\
11 & - & 1 \\
\hline
\end{tabular}

TPMT, thiopurine S-methyl transferase.

(Table 2). A possible explanation for this may be that a TPMT test is automatically ordered as part of routine laboratory orders for some physicians subspecializing in autoimmune diseases.

As expected, the all-time records review found a higher rate of DGT than analysis of the 2006 calendar year data alone. Of the 246 patients who had TPMT testing in 2006 included in the all-time records review, 10 had two or more tests in 2006 alone and seven additional patients had one test in 2006 and one or more additional tests at some time earlier than 2006. The alltime DGT rate most probably reflects the true incidence of DGT, but we were only able to determine the all-time rate for internal samples.

For internal patients having DGT, records were reviewed for an appropriate indication for repeating genetic testing. In no case was there documentation of an appropriate indication for DGT. From this, we conclude that appropriately indicated DGT is rare.

\section{System to limit duplicate HFE testing}

At the time of this study (2006), the laboratory performing HFE testing had a system in place to limit HFE DGT. During sample accession a technician identified requests for duplicate HFE testing through a computerized search of laboratory records. Duplicate tests continued through standard HFE test processing, while an attempt was made to contact the referring physician. Testing was canceled if the referring physician could be contacted and cancellation confirmed before HFE test completion.

In the consecutive series of 207 internal patients referred for $H F E$ testing, five of nine duplicate test requests were canceled using this protocol. For 2006, this system saved an estimated $\$ 77,479$ charges for duplicate HFE testing (95\% CI, \$35,512$184,015)$. This estimation was calculated by multiplying the percentage of canceled test requests per patient tested $(2.4 \%$; $95 \%$ CI, $1.1 \%-5.7 \%$, Wilson procedure) by the total number of patients being tested in 2006 (7851) and the charge for HFE testing. 


\section{Laboratory charges associated with DGT}

Laboratory charges for DGT identified in the 2006 data set were $\$ 76,728$. This was calculated using 2006 retail test charges (HFE \$411.20, TPMT \$289.10, CYP450 $2 D 6$ \$236.30). As we did not review $16 \%$ of TPMT tests and $46 \%$ of CYP450 2D6 tests or include DGT occurring outside of the 2006 calendar year data, the true charges associated with DGT in 2006 is higher than our estimate. This estimate also does not include the estimated $\$ 77,479$ in savings from the system preventing HFE DGT.

\section{DISCUSSION}

We found that DGT accounts for a small but measurable percentage of total testing volume for the studied tests. Our results should be generalizable to genetic testing in both academic and community medical practices. These results should also be generalizable to other high volume genetic tests such as Factor V Leiden, PT20210G->A, and cystic fibrosis mutation panel testing.

A body of literature has been published on inappropriate laboratory utilization, and DGT could be classified under this heading. A systematic review of clinical laboratory audits found $5 \%$ to $50 \%$ of laboratory testing could be classified as inappropriate. ${ }^{4}$ In the only published study of the appropriateness of a genetic test, $17 \%$ of APC tests for familial adenomatous polyposis were found to be requested for inappropriate indications. ${ }^{5}$ The only previous report of DGT was included in a study of genetic testing in liver transplant patients where seven duplicate genetic tests were identified in a cohort of 215 patients. ${ }^{6}$

We predict the incidence and costs associated with DGT will grow as the use of genetic testing continues to increase. In addition to limiting unnecessary testing and costs, another reason to limit DGT is that it is a potential source of medical errors. Clinicians may wait to implement needed treatment until the results of the genetic testing they ordered are available, when in fact the results are already available.

Limiting DGT at the laboratory level, as done by the HFE laboratory in this study, has several drawbacks. Even if the test is cancelled in the laboratory the costs of obtaining the sample, shipping, and the system to cancel the DGT remain. Although a laboratory-based system to eliminate DGT may decrease laboratory charges to the client, the cost of implementing such a system is borne by the laboratory. Also, any single laboratorybased system limiting DGT does not prevent DGT through sample submission to a different laboratory. For these reasons, our recommended approach to DGT would be a systemsbased approach to limit DGT at the time of test ordering. Ideally, a computerized test order entry system would be able to query laboratory databases and alert the ordering clinician to possible DGT. To our knowledge, such a system is not in operation anywhere. Another more feasible intervention would be increasing the accessibility of previous genetic testing results to ordering clinicians through existing medical records systems.

\section{ACKNOWLEDGMENTS}

Without the gracious assistance of many laboratory personnel this study would not have been possible. Harmeet Malhi, $\mathrm{MD}$, provided valuable editorial assistance.

\section{References}

1. Feder JN, Gnirke A, Thomas W, Tsuchihashi Z, et al. A novel MHC class I-like gene is mutated in patients with hereditary haemochromatosis. Nat Genet 1996;13:399-408.

2. Richard VS, Al-Ismail D, Salamat A. Should we test TPMT enzyme levels before starting azathioprine? Hematology 2007;12:359-360.

3. Vandel P, Talon JM, Haffen E, Sechter D. Pharmacogenetics and drug therapy in psychiatry-the role of the CYP2D6 polymorphism. Curr Pharm Des 2007;13:241250.

4. van Walraven $\mathrm{C}$, Naylor $\mathrm{CD}$. Do we know what inappropriate laboratory utilization is? A systemic review of laboratory clinical audits. JAMA 1998;280:550-558.

5. Giardiello FM, Brensinger JD, Petersen GM, Luce MC, et al. The use and interpretation of commercial APC gene testing for familial adenomatous polyposis. $N$ Engl JMed 1997;336:823-827.

6. Riegert-Johnson DL, Macaya D, Hefferon TW, Boardman LA. Medical errors related to genetic testing in liver transplant patients. Genet Test. In press. 